\title{
Root Asymptotics of Spectral Polynomials
}

\author{
B. Shapiro, M. Tater
}

We have been studying the asymptotic energy distribution of the algebraic part of the spectrum of the one-dimensional sextic anharmonic oscillator. We review some (both old and recent) results on the multiparameter spectral problem and show that our problem ranks among the degenerate cases of Heine-Stieltjes spectral problem, and we derive the density of the corresponding probability measure.

Keywords: Lamé operator, Van Vleck polynomials, asymptotic root-counting measure.

\section{QES sextic AHOs}

Our model is a 1D sextic anharmonic oscillator, which is frequently used to approximate various situations in quantum mechanics. Due to the quasi-exact solvability a part of the spectrum is easily tractable and two parameters of the potential leave enough space for the choice of application. To be more precise, we have been studying the solutions of the Schrödinger equation $H \psi=E \psi$, where $\psi \in L^{2}(\mathbb{R})$ and

$H=-\frac{\mathrm{d}^{2}}{\mathrm{~d} x^{2}}+x^{6}+b x^{4}+\left(\frac{b^{2}}{4}-(4 J+3)\right) x^{2}$

where $J$ is a non-negative integer. During the last decade there has been considerable interest in non-selfadjoint problems, e.g. [1], [2]. We recognize that $b \in \mathbb{C}$ is a challenge, but it goes beyond the scope of this paper, and we confine ourselves to $b \in \mathbb{R}$.

Adopting the idea of Bender and Dunne [3], we seek the solution $\psi$ of the Schrödinger equation $H \psi=E \psi$ in the form

$$
\psi(x)=e^{-\frac{x^{4}}{4}-\frac{b x^{2}}{4}} \sum_{n=0}^{\infty}\left(-\frac{1}{4}\right)^{n} \frac{P_{n}(E)}{n ! \Gamma\left(n+\frac{1}{2}\right)} x^{2 n}
$$

We are interested in even solutions only, and the odd ones can be treated by analogy. For $\psi$ to be a solution, the coefficients $P_{n}$ must fulfil the recurrence relation

$$
\begin{aligned}
P_{n}(E) & =\left(E-\frac{(4 n-3) b}{2}\right) P_{n-1}(E) \\
& +16(n-1)(n-J-2)\left(n-\frac{3}{2}\right) P_{n-2}(E)
\end{aligned}
$$

with the initial conditions $P_{-1}(E)=0$ and $P_{0}(E)=1$.

We see that the wave function $\psi$ serves as a generating function for the polynomials $P_{n}(E)$. Choosing a particular value of $J$ we get the particular solution of our problem, but for any non-negative value of $J$ the infinite system of $P_{n}$ forms an orthogonal system of monic polynomials with respect to a probability measure (Favard's theorem [4], [5]). The peculiarity is that the polynomials of degree $J$ or higher contain a common factor $P_{J-1}$, i.e. they can be factorized. The roots of the $P_{J}$ polynomial are the eigenvalues of the Schrödinger equation. These polynomials have other interesting properties [3], but we shall not use them.
The algebraic part of the spectrum, i.e. the quasi-exact energy eigenvalues, are solutions of $P_{J}(E)=0$ and the corresponding eigenfunctions are products

$$
\psi_{n}(x)=Q\left(x^{2}\right) \exp \left(T\left(x^{2}\right)\right) .
$$

We change the variable $x^{2} \rightarrow t$ and reduce the Schrödinger equation to

$4 t \frac{\mathrm{d}^{2} f}{\mathrm{~d} t^{2}}-\left(4 t^{2}+2 b t-2\right) \frac{\mathrm{d} f}{\mathrm{~d} t}+\left(4 J t+E-\frac{b}{2}\right) f=0$

We are thus faced by a linear differential operator and its polynomial solutions. This is a problem that has been studied by many prominent mathematicians since the first half of the $19^{\text {th }}$ century, and many nice results have been achieved.

\section{Heine-Stieltjes spectral problem}

Generally, a linear differential operator

$$
\mathcal{L}(z)=\sum_{i=1}^{k} Q_{i}(z) \frac{\mathrm{d}^{i}}{\mathrm{~d} z^{i}}
$$

with poynomial coefficients is called a (higher) Lamé operator (e.g. [6]). An important number

$$
r=\max _{i=1, \ldots, k}\left(\operatorname{deg} Q_{i}(z)-i\right)
$$

is called the Fuchs index of $\mathcal{L}(z)$. The case $r=0$ is called exactly solvable, and it has been well studied. These operators and their polynomial eigensolutions have many interesting properties. The operator $\mathcal{L}(z)$ is called non-degenerate if $\operatorname{deg} Q_{k}(z)=k+r$.

The multiparameter spectral problem

$$
\mathcal{L}(z) S(z)+V(z) S(z)=0,
$$

where a polynomial $V(z)$ of degree at most $r$ is sought so that the above equation will have a polynomial solution $S(z)$ of degree $n$. This is called the (higher) Heine-Stieltjes spectral problem, $V(z)$ a (higher) Van Vleck polynomial, and $S(z)$ a (higher) Stieltjes polynomial.

\subsection{Non-degenerate cases}

Two important results are worth mentioning at this point. First, a generalization of the result by Heine $(c f[6])$. 


\section{Theorem}

For any non-degenerate higher Lamé operator $\mathcal{L}(z)$ with algebraically independent coefficients of $Q_{i}(z), i=1, \ldots, k$ and for any $n \geq 1$ there exist exactly $\left(\begin{array}{c}n+r \\ n\end{array}\right)$ Van Vleck polynomials $V(z)$ 's and corresponding degree $n$ Stieltjes polynomials $S(z)$ 's.

Now, we mention also a physically important case

$\prod_{i=1}^{l}\left(z-\alpha_{i}\right) \frac{\mathrm{d}^{2} S}{\mathrm{~d} z^{2}}+\sum_{j=1}^{l} \beta_{j} \prod_{j \neq 1}\left(z-\alpha_{i}\right) \frac{\mathrm{d} S}{\mathrm{~d} z}+V S=0$

with $\alpha_{1}<\alpha_{2}<\ldots \alpha_{l}$ real and $\beta_{1}, \ldots, \beta_{l}$ positive. Then the following theorem holds.

Theorem (Stieltjes, Van Vleck, Bôcher, [6])

For any integer $n \geq 1$

1) there exist exactly $\left(\begin{array}{c}n+l-2 \\ n\end{array}\right)$ polynomials $V$ of degree $l-2$ such that equation (2) has a polynomial solution $S$ of degree exactly $n$.

2) each root of each $V$ and $S$ is real, simple, and belongs to the interval $\left(\alpha_{1}, \alpha_{l}\right)$.

3) none of the roots of $S$ can coincide with some of $\alpha_{i}$ 's. Moreover, $\left(\begin{array}{c}n+l-2 \\ n\end{array}\right)$ polynomials are in one-to-one correspondence with $\left(\begin{array}{c}n+l-2 \\ n\end{array}\right)$ possible ways to distribute $n$ points into $l-1$ open intervals $\left(\alpha_{1}, \alpha_{2}\right),\left(\alpha_{2}, \alpha_{3}\right), \ldots,\left(\alpha_{l-1}, \alpha_{l}\right)$.

Recently, Borcea and Shapiro [7] found the density of the asymptotic root distribution for the Stieltjes polynomials.

\subsection{The degenerate case}

Inspecting the structure of (1) we see that it is a degenerate case of the $r=1$ operator. The roots of Van Vleck polynomials are not confined to a finite interval as $n \rightarrow \infty$. In order to find their limitng distribution we have to scale them. To this end we must know their rate of growth.

$$
M_{n}:=\left(\begin{array}{ccccccc}
E-\xi_{n, 1} & \alpha_{n, 2} & 0 & 0 & \cdots & 0 & 0 \\
\gamma_{n, 2} & E-\xi_{n, 2} & \alpha_{n, 3} & 0 & \cdots & 0 & 0 \\
0 & \gamma_{n, 3} & E-\xi_{n, 3} & \alpha_{n, 4} & \cdots & 0 & 0 \\
\vdots & \vdots & \ddots & \ddots & \ddots & \vdots & \vdots \\
0 & 0 & 0 & \ddots & \ddots & \alpha_{n, n-1} & 0 \\
0 & 0 & 0 & \cdots & \gamma_{n, n-1} & E-\xi_{n-1, n-1} & \alpha_{n, n} \\
0 & 0 & 0 & \cdots & 0 & \gamma_{n, n} & E-\xi_{n, n}
\end{array}\right)
$$

and

$$
\xi_{n, i}=\frac{4 n-4 i+1}{2 C_{n}}, \quad \quad \alpha_{n, i}=\frac{4(i-1)}{C_{n}}
$$$$
\gamma_{n, i}=\frac{(2 n-2 i+1)(2 n-2 i+2)}{C_{n}}
$$
method. Let

$$
P_{n}(E)=E^{n}+\sum_{l=0}^{n-1} p_{l} E^{l}
$$
roots

$$
s_{k}=E_{1}^{k}+\cdots+E_{n}^{k} .
$$

$$
\lim _{k \rightarrow \infty} \frac{s_{k}}{E_{\max }^{k}}=1 .
$$

Because $s_{k}$ are related to $p_{l}$ by

$$
s_{k}+\sum_{l=1}^{k-1} p_{n-l} s_{k-l}+k p_{n-k}=0
$$
formulae can be derived from the recurrence relation.

We arrive at

$$
s_{k}=C(k) b^{\varepsilon} n^{\kappa}+\mathcal{O}\left(n^{\kappa-1}\right)
$$

where

and $l=0,1,2, \ldots$. Thus,

$$
\lim _{k \rightarrow \infty} \sqrt[k]{C(k)}=\pi
$$
tridiagonal matrix $S p_{n}=\operatorname{det}\left(M_{n}\right)$, where

Thus, we are interested in the roots of the maximal modulus and their dependence on parameter $b$. To find this dependence we follow the main idea of the Gräffe-Lobachevskii

and we find expressions for the sums of the powers of the

Then for the root of the maximal modulus $E_{\max }$ holds

we need explicit expressions for $p_{l}$ up to $l=n-k$. These

$$
\left\{\begin{array}{cccc}
\kappa=3 l+2 & \& & \varepsilon=1 & \text { if } k=2 l+1 \\
\kappa=3 l+4 & \& & \varepsilon=0 & \text { if } k=2 l+2
\end{array}\right.
$$

$$
\lim _{k \rightarrow \infty} \sqrt[k]{s_{k}}=\lim _{k \rightarrow \infty} \sqrt[k]{C(k)} b^{\frac{\varepsilon}{k}} n^{\frac{\kappa}{k}}=n^{\frac{3}{2}} \lim _{k \rightarrow \infty} \sqrt[k]{C(k)}
$$

i.e. the limit is independent of $b$. Our conjecture is that

Polynomials $S p_{n}$ that have the same roots as $P_{n}$ but scaled by $C_{n}=C n^{\frac{3}{2}}$ do not fulfil any finite recurrence relation. However, we can rewrite them as a determinant of an $n \times n$ 
This enables us to extend the polynomial sequence by introducing a subsequence $S p_{n, i}=\operatorname{det}\left(M_{n, i}\right)$, where $M_{n, i}$ is the upper $i \times i$ principal submatrix of $M_{n}$ and this enables us to use the result of Kuijlaars and Van Assche concerning the three-term recurrence relations with variable coefficients [8]. This relation reads

$$
S p_{n, i}(E)=\left(E-\xi_{n, i}\right) S p_{n, i-1}-\alpha_{n, i} \gamma_{n, i} S p_{n, i-2}
$$

with initial conditions $S p_{n, 0}=1$ and $S p_{n,-1}=0$. Now, it remains to go to the polynomials $T p_{n, i}$ that have the same roots as $S p_{n, i}$ and fulfil the relation

with

$$
E T p_{n, i-1}=a_{n, i+1} T p_{n, i}+\xi_{n, i} T p_{n, i-1}+a_{n, i} T p_{n, i-2},
$$

$$
a_{n, i}=\sqrt{\frac{4(i-1)(2 n-2 i+1)}{C_{n}}}
$$

The limits

$\xi(\tau)=\lim _{i / n \rightarrow \tau} \xi_{n, i}=0, a(\tau)=\lim _{i / n \rightarrow \tau} a_{n, i}=4 \sqrt{\frac{\tau(\tau-1)^{2}}{C_{n}}}$

determine the density of the asymptotic root-counting measure:

$$
\int_{0}^{1} \omega_{[-2 a(\tau), 2 a(\tau)]} \mathrm{d} \tau
$$

where

$$
\omega_{[x, y]}=\left\{\begin{array}{cl}
\frac{1}{\pi \sqrt{(y-s)(s-x)}} & \text { if } s \in[x, y] \\
0 & \text { otherwise }
\end{array}\right.
$$

The sought density $\rho$ is then expressed as

$$
\frac{C}{\pi} \int_{0}^{1} \frac{\mathrm{d} \tau}{\sqrt{64 \tau(\tau-1)^{2}-C^{2} s^{2}}} \text {. }
$$

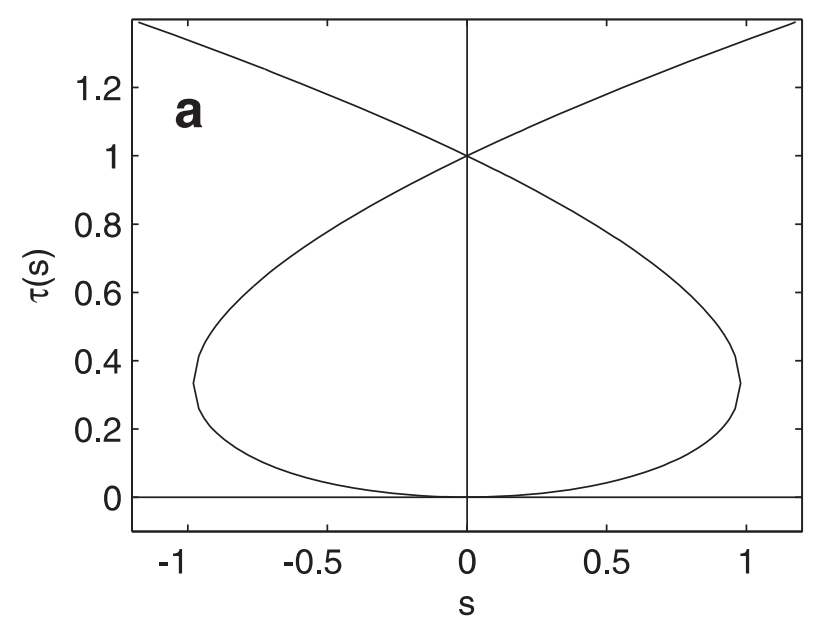

We immediately see that the integral does not converge for $s=0$. The polynomial under the square root has three real roots $\tau_{1}(s)<\tau_{2}(s)<\tau_{3}(s)$ if $s \in\left[-\frac{16}{3 \sqrt{3} C}, \frac{16}{3 \sqrt{3} C}\right]$ and $\tau_{3} \geq 1$ for
all $s \in \mathbb{R}$. Thus $\rho$ can be written as

$$
\rho=\frac{C}{\pi} \int_{\tau_{1}}^{\tau_{2}} \frac{\mathrm{d} \tau}{\sqrt{64\left(\tau-\tau_{1}\right)\left(\tau_{2}-\tau\right)\left(\tau_{3}-\tau\right)}}
$$

which can be evaluated as [9]

$\rho(s)=\frac{C}{8} \frac{1}{\sqrt{\tau_{3}-\tau_{1}}}{ }_{2} F_{1}\left(\frac{1}{2}, \frac{1}{2}, 1, \frac{\tau_{2}-\tau_{1}}{\tau_{3}-\tau_{1}}\right) \quad s \in\left[-\frac{16}{3 \sqrt{3} C}, \frac{16}{3 \sqrt{3} C}\right]$.

\section{Acknowledgments}

This research was partially supported by project LC06002.

\section{References}

[1] Shin, K. C.: Schrödinger Type Eigenvalue Problems with Polynomial Potentials: Asymptotics of Eigenvalues, math. $\mathrm{SP} / 0411143$.

[2] Trinh, D. T.: Asymptotique et analyse spectral de l'oscillateur cubique, Th se, Université de Nice, 2002.

[3] Bender, C. M., Dunne, G. V.: Quasi-Exactly Solvable Systems and Orthogonal Polynomials, J. Math. Phys., Vol. 37 (1996), p. 6-11.

[4] Chihara, T. S.: An Introduction to Orthogonal Polynomials. Gordon and Breach, New York, 1978.

[5] Marcellán, F., Álvarez-Nodarse, R.: On the "Favard's Theorem" and its Extensions. J. Comp. Appl. Math., Vol. 127 (2001), p. 231-254.

[6] Borcea, J., Brändén, P., Shapiro, B.: Higher Lamé Equations, Heine-Stieltjes and Van Vleck Polynomials, in preparation.

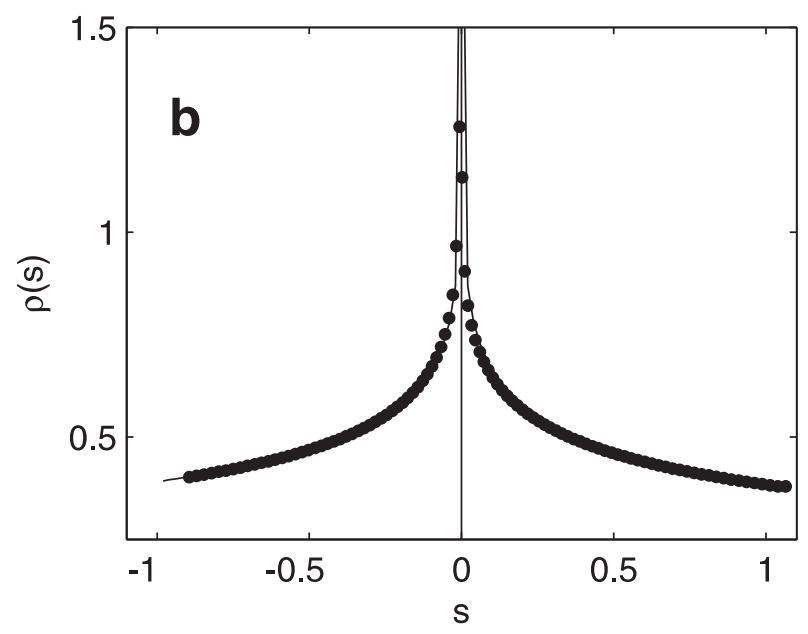

Fig. 1: a) Dependence of the three real roots of $64 \tau(\tau-1)^{2}-C^{2} s^{2}=0$ on $s$ (for $C=\pi$ ). If $s \notin\left[-\frac{16}{3 \sqrt{3} C}, \frac{16}{3 \sqrt{3} C}\right]$, there is only one real root $\tau_{3}(s)>1$; b) Comparison of density $\rho$ with a numerical example for $n=100$ and $b=2.66$. 
[7] Borcea, J., Shapiro, B.: Root Asymptotics of Spectral Polynomials for the Lamé Polynomials, math. CA/0701883.

[8] Kuijlaars, A. B. J., Van Assche, W.: The Asymptotic Zero Distribution of Orthogonal Polynomials with Varying Recurrence Coefficients, J. Approx. Theory, Vol. 99 (1999), p. 167-197.

[9] Prudnikov, A. P., Brychkov, Yu. A., Marichev, O. I.: Integrals and Series. Elementary Functions. Nauka, Moscow, 1981.

Prof. Boris Shapiro

e-mail: shapiro@mat.su.se

Department of Mathematics

University of Stockholm

S-10691 Stockholm, Sweden

RNDr. Miloš Tater, CSc.

e-mail: tater@ujf.cas.cz

Nuclear Physics Institute, Academy of Sciences

CZ-25068 Řež near Prague 